\title{
Alteraciones de la altura tonal e intensidad vocal en niños de 4 a 6 años de las instituciones de educación inicial públicas del distrito de Magdalena del Mar en el año 2015.
}

Alterations of the tonal height and vocal intensity in children from 4 to 6 years old of the public institutions of initial education in Magdalena del Mar district in 2015

Maria Esperanza Márquez Flores ${ }^{1}$, Hanna Fátima Del Solansh Saldaña Tapia ${ }^{1}$, Stefani Luisa Vicente Bustillos $^{1}$, Paul Rubén Alfaro Fernández ${ }^{1}$, Milagros Rojas Llerena ${ }^{1}$.

\section{RESUMEN}

A lo largo del tiempo, las alteraciones de voz han incrementado su presencia significativamente, más aún en la población infantil, afectando muchas veces su comunicación y desarrollo personal, por lo que es importante generar una cultura de prevención. Objetivo: Determinar la frecuencia de las alteraciones de la altura tonal e intensidad vocal en niños de 4 a 6 años de las Instituciones de Educación Inicial Públicas del Distrito de Magdalena del Mar en el año 2015. Material y Métodos: Estudio descriptivo de tipo transversal. Se utilizaron dos instrumentos para la recolección de datos: Una Ficha de Recolección que fue respondida por los padres de familia sobre el género, la edad y el estado actual de la voz de su niño y un Software de análisis acústico PRAAT (Versión 5.4.06) con el cual se midió la altura tonal e intensidad vocal, permitiendo determinar si la voz era la adecuada o presentaba alguna alteración, al ser comparados los resultados obtenidos con los parámetros bibliográficamente establecidos. Resultados: De una muestra total de 128 niños, se recolectó 117 datos, el 22,22\% de esta población presentó tanto la altura tonal como la intensidad vocal alterados, donde el 65,38\% fueron de género femenino. Además los niños de 4 y 6 años presentaron su altura tonal alterada, con un 34,29\% a su población, proporcionalmente. Conclusiones: El $55,56 \%$ de la muestra presentó una alteración a nivel de la intensidad vocal, lo que indica un inadecuado uso vocal.

PALABRAS CLAVE: Voz, altura tonal, intensidad vocal.

\section{SUMMARY}

Over time, the voice alterations have increased their presence significantly, affecting frequently the communication and personal development of the child population. For this reason is important to generate a culture of prevention. Objective: To determinate the frequency of the alterations of the tonal height and vocal intensity in children from 4 to 6 years old of the public Institutions of Initial Education in Magdalena del Mar district in 2015. Material and Methods: Descriptive study of transverse type. Two instruments were used for data collection: A record collection sheet that was answered by parents about the gender, age and the actual state of the voice of their child. Also a software of acoustic analysis PRAAT (Version 5.4.06) which was used to measure the tonal height and vocal intensity. It allowed to determinate if the voice was suitable or presented any alteration when the results obtained were compared with the bibliographitly parameters established. Results: A total sample of 128 children was collected 117 
data, the $22.22 \%$ of this population presented both the tonal height and the vocal intensity altered, where $65.38 \%$ was of female gender. Also children of 4 and 6 years old presented their pitch altered this represents a $34.29 \%$ of their population proportionately. Conclusions: The $55.56 \%$ of the sample presented an alteration of the level of the vocal intensity which indicated a vocal inappropriate use.

KEY WORDS: Voice, pitch/ tonal height, vocal intensity.

\section{INTRODUCCIÓN}

A lo largo de los años las alteraciones de voz han incrementado su presencia, afectando a millones de personas a lo largo del mundo. La mayor parte de estudios epidemiológicos refiere que entre el 6 y $37,14 \%$ de la población infantil presentan alteraciones vocales; las cuales afectan de forma negativa, su comunicación y desarrollo personal (1).

Entre las patologías benignas más frecuentes encontramos a la disfonía infantil, la cual tiene etiología variada, pudiendo estar relacionada a factores orgánicos, conductuales y emocionales (2).

Azevedo refiere que dicha patología está relacionada con el abuso y mal uso vocal. Esa relación se refiere a la producción vocal inadecuada, pudiéndose destacar un habla con esfuerzo y exceso de tensión, carraspeo $\mathrm{y}$ variaciones en las cualidades de la voz, que pueden ser características comunes en niños que la desarrollan (3).

La cualidad vocal, es una definición usada para describir las características de la voz. En el niño ésta se encuentra determinada por el ambiente: social, educativo, factor hereditario, psicológico y fisiológico; incluyendo los factores de: edad, género, salud, estructura del aparato fonador, funcionamiento de la laringe y la caja de resonancia (4).

Las cualidades vocales permiten identificar alteraciones en la voz, para ello es necesario evaluar la misma determinando el tipo de voz, la resonancia, la frecuencia fundamental y la intensidad vocal (4).

La frecuencia fundamental, también conocida como altura tonal o pitch, es una frecuencia de la vibración de los pliegues vocales. Estas frecuencias y sus valores son fundamentales tanto para hombres, mujeres y niños y se expresa de forma numérica, en Hertz (5).
Existen cambios en la Frecuencia fundamental a lo largo de la vida del individuo. En ambos géneros la frecuencia es parecida hasta la pubertad, durante la cual ocurre un descenso de laringe en varones, dando como resultado una voz grave, mientras que en la mujeres se mantiene (6).

De acuerdo a Capperali, la altura tonal normal en niños de cuatro años se encuentra desde 266,6 a $375 \mathrm{~Hz}$ para mujeres, y de 285,6 a $355 \mathrm{~Hz}$ para hombres; en niños de cinco años se encuentra desde 247.5 a 350 $\mathrm{Hz}$ para hombres y de 247,6 a $355 \mathrm{~Hz}$ para mujeres; y finalmente en niños de seis años se encuentra desde 247,4 a $325 \mathrm{~Hz}$ para hombres y de 247,0 a $315 \mathrm{~Hz}$ para mujeres (7).

La frecuencia fundamental puede ser medida con instrumentos sofisticados de laboratorio, instrumentos computarizados, además de pruebas sugestivas (5). Por su parte, la intensidad vocal, también conocida como Loudness, es el producto de la amplitud de vibración de las cuerdas vocales y de la presión subglótica, la cual se expresa en decibelios (dB) (5).

La intensidad puede evaluarse mediante la observación dentro de una conversación con el individuo, en diversas actividades, como: dar clase a aulas, locución, canto, etc. Incluyendo, los abusos vocales que puede realizar (4).

Teixeira, refiere que los valores normativos de la intensidad vocal para niños en cuanto a la emisión prolongada de la vocal "a" varía de los 69,7 a 78,9 dB (8).

Países como Estados Unidos, España, Brasil, junto a algunos estados de Latinoamérica, han realizado algunas investigaciones sobre las cualidades de la voz en pacientes con o sin alteraciones de la misma, utilizando programas como el ANAGRAF, PRAAT o VoxMetría; programas de los cuales se obtienen registros con datos objetivos sobre los parámetros de la voz, los cuales brindan soporte físico de los hallazgos subjetivos (9). 
Los estudios de la voz requieren complementarse con el análisis de medidas objetivas que ofrezcan datos cuantitativos sobre el nivel de perturbación de la frecuencia y de la intensidad, la presencia o ausencia de ruido y su inestabilidad. Estos están sujetos a la variabilidad según la edad y el sexo de las personas, debido a las diferencias fisiológicas y anatómicas de las estructuras glóticas y supraglóticas (10).

Poder contar con perfiles de normalidad de los parámetros acústicos de la voz, permite conocer objetivamente los cambios en frecuencia e intensidad de las emisiones vocales, visualizándose mediante una gráfica o por medio de una cifra las principales características de las ondas sonoras producto de la voz. Esto ha resultado una alternativa importante para el campo de la fonoaudiología (11).

Según Monteiro el parámetro acústico es un método cuantitativo, no invasivo de evaluación de la voz el cual ofrece una evaluación objetiva y eficiente (8).

En el Perú, no existen estudios objetivos relacionados a las cualidades de la voz y mucho menos se le da la importancia a sus alteraciones.

Doctores como Ignacio Cobeta y Antonio Martín, otorrinos de especialidad, recalcan sobre la necesidad de cuidar la voz por ser nuestro instrumento de comunicación. Por ello en caso de presentarse alteraciones en la voz con una duración mayor a quince días hay una necesidad de realizar una evaluación especializada (12).

Asimismo Devars resalta que la asistencia tardía al otorrinolaringólogo provoca que en las disfonías funcionales sean muy frecuentes las lesiones orgánicas (13).

Vila, nos afirma que los niños aprenden modelos vocálicos presentes en su entorno: medios de comunicación, juegos electrónicos; los cuales al ser visualizados son imitados exagerando características vocales, pudiendo esto finalmente ser perjudicial para la voz de los niños (14). Le Huche y Allali nos afirman que estos problemas generalmente, se constituyen hacia los seis o siete años de edad (15).

Dinville, estima que las disfonías infantiles afectan a cerca de la mitad de los niños en edad escolar, desde los cinco o seis años hasta la adolescencia
(16); y Cooper, resalta el desconocimiento de esta problemática en la sociedad (17).

En el ámbito nacional, Unda, en el Distrito de SurcoLima, realizó un estudio descriptivo con 40 niños entre 6 y 7 años con el objetivo de determinar las Incidencias de disfonías en los alumnos del primer grado del Colegio Particular Cristo Salvador, empleando un cuestionario adaptado por CPAL y el Protocolo de Evaluación de voz de Mara Behlau; encontrando que el $10 \%$ de la muestra presentó esfuerzo vocal, como causa principal de la disfonía funcional; además se evidenciaron periodos cortos de enfermedades que podrían repercutir en las alteraciones de la voz, siendo: el $12,5 \%$ tensión muscular en el cuello y el $2,5 \%$ resonancia laringofaríngea (18).

En comparación con otros países como España y Brasil, en donde se puede encontrar diverso material que aporte información importante. Se tiene como referencia a Borragán et al., con una muestra de 919 niños de la Comunidad Autónoma de Cantabria y mediante el uso de escala de GRBAS detectaron que el porcentaje total de niños que presentaban trastornos en la voz era del $36 \%$ (19).

Colombo realizó un estudio de tipo exploratorio de diseño transversal con una población de 83 niños sobre la prevalencia de disfonía infantil en niños de 8 a 12 años de ambos sexos, empleando un Protocolo de Evaluación del Proceso Fonatorio, concluyendo que la disfonía infantil está presente en 11 de los niños investigados $(13,2 \%)$, predominando en varones $(81,8 \%)$ sobre $(18,2 \%)$ mujeres y siendo más frecuente en niños de 12 años (20).

Feijó determinó que la prevalencia de disfonía infantil varió entre $6 \%$ a $37,14 \%$ en los estudios revisados, siendo la aplicación de cuestionarios y la evaluación perceptivo auditiva las más utilizadas (21).

Por tal motivo y teniendo en consideración que no se han realizado estudios relacionados a las cualidades de la voz en los niños en el Perú de manera objetiva, nuestro estudio pretende realizar la medición de las cualidades de la voz como la altura tonal e intensidad vocal objetivamente mediante un análisis acústico (Programa PRAAT).

Lapresenteinvestigaciónes devitalimportanciapara la población infantil, ya que los resultados obtenidos 
servirán como precedente para investigaciones futuras que busquen prevenir complicaciones que puedan afectar el tracto vocal a largo plazo, generando así una cultura de prevención necesaria para que los niños logren un óptimo desarrollo.

Asimismo, a nivel teórico tanto nuestros resultados como la revisión bibliográfica servirán para incrementar el cuerpo teórico existente en nuestro medio acerca de esta temática tan poco conocida.

El objetivo de la presente investigación fue determinar la frecuencia de alteraciones de altura tonal e intensidad vocal según género y edad, identificando la relación con las variables intervinientes como: Enfermedades respiratorias,voz ronca, habla en volumen muy alto o fuerte y tiempo de ronquera.

\section{MATERIAL Y MÉTODOS}

El distrito seleccionado se caracteriza por un clima húmedo constante, el cual podría favorecer que los niños presenten diversas características vocales.

El tipo de estudio que se ajusta a la presente investigación es descriptivo de tipo transversal. Mediante el programa Openepi Versión 3.03 se obtuvo una muestra de 128 niños de una población total de 454.

Se utilizó un muestreo probabilístico por el método estratificado proporcional, dentro de los criterios de inclusión se encuentran los niños matriculados en las instituciones; por otro lado, se excluyeron aquellos niños cuyos padres rechazaron firmar el consentimiento informado, así como los que formaban parte del programa de inclusión y que sus edades no se encontraban entre los 4 y 6 años de edad.

Para la selección de participantes se solicitó previamente a la institución el listado de estudiantes matriculados. Para participar en el estudio se invitó a los padres de los estudiantes matriculados a una charla explicativa sobre el problema de la tesis y su importancia en el medio, además se les entregó un consentimiento informado de manera personal donde se especificó: el propósito del estudio, recolección de datos, riesgos, beneficios, costos e incentivos, confidencialidad y derechos del paciente. Con el fin, de darle la oportunidad de aceptar o rechazar el estudio mediante la firma del apoderado. Asimismo, se le especificó al padre de familia que recibiría un informe de evaluación si éste aceptaba la participación del menor en el estudio.

En el proceso de recolección de datos se utilizaron dos instrumentos: Una Ficha de Recolección, que fue aprobada por criterio de jueces y respondida por los padres de familia sobre el género, la edad y el estado actual de la voz de su niño desde su percepción personal.

El segundo instrumento utilizado fue el Software de análisis acústico PRAAT (Versión 5.4.06); el cual para demostrar su confiabilidad: se han realizado estudios para comparar su funcionalidad, como: Núñez Batalla Faustino (22), quien correlacionó los resultados en dos ámbitos: Espectrograma de banda estrecha y Parámetros acústicos de la voz, en 99 muestras de voz diagnosticadas mediante videoestroboscopía de edema de Reinke, con el programa Dr. Speech y el programa Praat, y se concluyó que ambos softwares ofrecen similares resultados en el análisis acústico de las voces patológicas (22).

Por otro lado, se utilizó un audífono con micrófono incorporado, con soporte para cabeza modelo H-MIC Detachable $3.5 \mathrm{~mm}$ Neckband Headset maxell; tipo omnidireccional, con una respuesta de frecuencia de $20-15 \mathrm{kHz}$, sensibilidad $-60+/-3 \mathrm{~dB}$ e impedancia de salida máxima de $2.2 \mathrm{~K}$ Ohmio situado a $10 \mathrm{~cm}$ de la boca (23).

La realización del presente estudio, se realizó en un ambiente aislado de ruido y de manera individual, donde se le solicitó al menor sentarse adecuadamente en una silla y se procedió a colocarle el auricular con micrófono incorporado a una distancia de $10 \mathrm{~cm}$ de la boca, con el fin de indicarle que pronuncie la vocal "a" sostenida por 7 segundos.

Cabe señalar que se realizaron de tres a cuatro pruebas previas al análisis, con el fin de que el niño comprenda la consigna antes de obtener los resultados finales necesarios para el estudio.

Este proceso fue realizado para ambas cualidades, frecuencia fundamental e intensidad vocal, permitiéndonos de esta manera determinar si la voz es adecuada o presenta alguna alteración, al ser comparados los resultados con los parámetros bibliográficamente establecidos.

Finalmente, se le informó a los padres de manera personal y confidencial de los resultados que se 
obtuvieron en el presente estudio mediante un informe, el cual fue presentado al final del término de las evaluaciones tomadas a todos los niños que participaron en el estudio, donde de ser necesario, fueron derivados hacia un especialista.

\section{RESULTADOS}

De una muestra total de 128 niños, únicamente se recolectaron 117 datos debido a que no todos los padres aceptaron que sus menores hijos participasen en el estudio; además, a pesar de que se seleccionaron nuevamente a otros niños, no todos asistieron.

Para establecer la frecuencia según los objetivos se utilizó el programa Stata Versión 13, describiendo las variables y utilizando el método de chi cuadrado para relacionar las variables principales con las secundarias. Dentro de los resultados obtenidos podemos observar que: De 117 niños; el 23,08\% tienen 4 años, el 53,85\% tienen 5 años y el 23,08\% restante tienen 6 años. Siendo el $52,14 \%$ de género femenino, y el $47,86 \%$ de género masculino (tablas 1 y 2).

El $55,56 \%$ de la muestra presentó la intensidad vocal alterada, mientras un $29,91 \%$ presentó la altura tonal alterada. Por otro lado, el $22.22 \%$ de la

Tabla 1. Relación de las variables principales con las secundarias.

\begin{tabular}{cc}
\hline Edad & Frecuencia (\%) \\
\hline 4 & 23,08 \\
5 & 53,85 \\
6 & 23,08 \\
Total & 100,00 \\
\hline
\end{tabular}

Tabla 2. Relación de las variables principales con las secundarias.

\begin{tabular}{cc}
\hline Genero & Frecuencia (\%) \\
\hline Femenino & 52,14 \\
Masculino & 47,86 \\
Total & 100,00 \\
\hline
\end{tabular}

población, presentaron ambas cualidades alteradas (tabla 3).

Al relacionar estos últimos resultados con las variables intervinientes como: Enfermedades respiratorias, voz ronca, habla en volumen muy alto o fuerte y tiempo de ronquera no se encontraron diferencias estadísticamente significativas.

Tabla 4. Alteración de la altura tonal e intensidad vocal relacionado a las enfermedades respiratorias.

\begin{tabular}{lcc}
\hline Ambos & \multicolumn{2}{c}{$\begin{array}{c}\text { Enfermedades } \\
\text { respiratorias }\end{array}$} \\
\hline & Si (\%) & No (\%) \\
Alterado & 20,97 & 23,64 \\
No alterado & 79,03 & 76,36 \\
Total & 100 & 100 \\
Respuesta por parte de los padres & 52,99 & 47,01 \\
\hline $\mathrm{Pr}=0,729$ & &
\end{tabular}

Tabla 5. Alteración de altura tonal e intensidad vocal relacionado al grito en el habla.

\begin{tabular}{lcc}
\hline Ambos & \multicolumn{2}{c}{ Grito en habla } \\
\hline & $\mathrm{Si}(\%)$ & No (\%) \\
Alterado & 17,78 & 25 \\
No alterado & 82,22 & 75 \\
Total & 100 & 100 \\
Respuesta por parte de los padres & 38,46 & 61,54 \\
\hline $\mathrm{Pr}=0,361$ & &
\end{tabular}

Tabla 6. Alteración de altura tonal e intensidad vocal relacionado a la voz ronca.

\begin{tabular}{lcc}
\hline Ambos & \multicolumn{2}{c}{ Voz ronca } \\
& Si (\%) & No (\%) \\
\hline Alterado & 33,33 & 25 \\
No alterado & 66,67 & 75 \\
Total & 100 & 100 \\
Respuesta por parte de los padres & 10,26 & 89,74 \\
\hline $\mathrm{Pr}=0,535$ & &
\end{tabular}

Tabla 3. Alteración de altura tonal e intensidad vocal.

\begin{tabular}{lccc}
\hline Cualidades & Alterado (\%) & No alterado (\%) & Total (\%) \\
\hline Altura tonal & 29,91 & 70,09 & 100 \\
Intensidad vocal & 47,86 & 44,44 & 100 \\
Ambos & 100,00 & 77,70 & 100 \\
\hline
\end{tabular}


Tabla 7. Alteración de altura tonal e intensidad vocal relacionado al tiempo de ronquera.

\begin{tabular}{lccccc}
\hline \multirow{2}{*}{ Ambos } & \multicolumn{5}{c}{ Tiempo de ronquera (\%) } \\
\cline { 2 - 6 } & Ninguna semana & 1 semana & 2 semana & 3 semana & 4 semana \\
\hline Alterado & 21,15 & 0 & 33,33 & 33,33 & 50 \\
No alterado & 78,85 & 100 & 66,67 & 66,67 & 50 \\
Total & 100 & 100 & 100 & 100 & 100 \\
Respuesta por parte de los padres & 88,89 & 2,56 & 2,56 & 2,56 & 3,42 \\
\hline
\end{tabular}

Por su parte, del $47,01 \%$ de aquellos padres que indicaron que sus hijos no presentaban enfermedades respiratorias, el $23,64 \%$ presentaron la altura tonal e intensidad vocal alterados (tabla 4).

Del $61,54 \%$, de aquellos padres que indicaron que sus hijos no gritan al hablar, el $25 \%$ presentaron la altura tonal e intensidad vocal alterados (tabla 5).

Del $89,74 \%$, de aquellos padres que indicaron que sus hijos no presentaban la voz ronca, el20,95\% presentaron la altura tonal e intensidad vocal alterados (tabla 6).

Del $88,89 \%$, de aquellos padres que indicaron que sus hijos no presentaban ronquera por ninguna o más semanas, el $21,15 \%$ presentaron la altura tonal e intensidad vocal alterados (tabla 7).

Tabla 8. Alteración de altura tonal según la edad.

\begin{tabular}{lcc}
\hline \multirow{2}{*}{ Edad } & \multicolumn{2}{c}{ Altura tonal (\%) } \\
\cline { 2 - 3 } & alterados & No alterados \\
\hline 4 años & 34,29 & 18,29 \\
5 años & 31,43 & 63,41 \\
6 años & 34,29 & 18,29 \\
Total & 100 & 100 \\
Altura de altura tonal & 29,91 & 70,09 \\
\hline
\end{tabular}

Tabla 9. Alteración de altura e intensidad vocal según el género.

\begin{tabular}{lcc}
\hline Genero & \multicolumn{2}{c}{ Ambos (\%) } \\
\cline { 2 - 3 } & Alterado & No alterado \\
\hline Femenino & 65,38 & 48,35 \\
Masculino & 34,62 & 51,65 \\
Total & 100 & 100 \\
Alteración de altura tonal e & 22,22 & 77,78 \\
intensidad vocal & \multicolumn{2}{c}{} \\
\hline
\end{tabular}

$\operatorname{Pr}=0,125$
$\mathrm{Al}$ relacionar la población que presenta la altura tonal alterada con la edad, se encontró un pr de 0,006 , existiendo diferencias estadísticamente significativas, lo cual indica que los niños de 4 y 6 años presentan el $34,29 \%$ la altura tonal alterada (tabla 8).

No obstante, del $22,22 \%$ de los menores que presentaron ambas cualidades alteradas. E165,38\% fueron de género femenino, sin existir diferencias estadísticamente significativas (tabla 9).

\section{DISCUSIÓN}

En relación a los materiales y métodos utilizados, se observó que Colombo (1999) utilizó como instrumento de medición el Protocolo de Evaluación del Proceso Fonatorio. Borragán, Barrio, Guisández y Fernández (1999), por su parte, emplearon la escala de GRBAS (Escala de Evaluación Auditiva- Perceptiva de la voz) para la realización de su estudio y Unda (2013), realizó su estudio mediante un cuestionario adaptado por CPAL y el Protocolo de Evaluación de voz de Mara Behlau.

Feijó, años más tarde, concluyó que en la mayoría de estudios revisados se aplicaron cuestionarios y evaluaciones perceptivo auditiva, instrumentos los cuales medían las características vocales de manera subjetiva, pudiendo dar un margen de error en comparación a los resultados del presente estudio (21). En relación a los tipos de estudio utilizados, se tiene que Colombo y Borragán et al., utilizaron estudios exploratorios de tipo transversal $(19,20)$, y Unda por su parte, utilizó un tipo de estudio descriptivo simple (18), a diferencia del presente estudio en el cual se utilizó un tipo de estudio descriptivo transversal, ya que permitía describir las variables y analizar su frecuencia e interrelación en un momento determinado. Respecto a los resultados obtenidos del presente estudio, éstos demostraron que existe mayor prevalencia de alteraciones de altura tonal e intensidad vocal en mujeres, a diferencia de lo que la literatura refiere. Por otro lado, el presente estudio demostró que 
el $22,22 \%$ de los niños evaluados entre cuatro y seis años presentaron ambas cualidades alteradas. Dinville estimó que las disfonías infantiles afectan a cerca de la mitad de los niños en edad escolar, desde los cinco o seis años hasta la adolescencia (16).

Feijó determinó que la prevalencia de disfonía infantil varió entre $6 \%$ a $37,14 \%$ en los estudios revisados (21). El presente estudio demostró que el $22,22 \%$ de la muestra presentó ambas cualidades vocales alteradas, porcentaje el cual se encontró dentro del parámetro revisado por Feijó.

El estudio mostró que existe un gran número de padres de familia que refieren que la voz de su niño no se encontraba alterada, sin embargo los resultados obtenidos del presente estudio mostraron lo contrario. Cooper por su parte, refirió que existe desconocimiento de las disfonías en la sociedad (17).

\section{Correspondencia}

Paul Rubén Alfaro Fernández

Correo electrónico: paul.alfaro.f@upch.pe

\section{REFERENCIAS BIBLIOGRÁFICAS}

1. Carding P, Roulstone S, Northstone, K. The ALSPAC Study Team. The prevalence of childhood dysphonia: a cross-sectional study. Journal of Voice. 2006; 20(4): 623-630. Doi: https://doi.org/10.1016/j. jvoice.2005.07.004

2. Freitas M, Weckx R, Pontes L. Disfonia na infância. Revista Brasileira de Otorrinolaringologia. 2000; 66(3): 257-265. (Citado el 13 de mayo del 2015) Disponible en: http://oldfiles.bjorl.org/conteudo/ acervo/acervo.asp?id $=2451$

3. Azevedo R. Disfonia na Infância. Tratado de Fonoaudiologia. Roca: Ed. São Paulo;2010.

4. Behlau M, Pontes P. Avaliação e tratamento das disfonias. Rio de janeiro: Lovise;1995.

5. Behlau M. El libro de la especialista. Rio de Janeiro: Revinter; 2001.

6. Casado J. Enfermedades del oído. Marbella: Sociedad Española de otorrinolaringología y patología cervicofacial; 2012.

7. Capperali V. Características acústicas de la voz en niños pre-escolares. [Internet]; Rev Bras Otorrinolaringol. 2008;74(2):265-72. (Citado el 8 de Junio del 2015) Disponible en: http://www.redalyc. org/articulo.oa? id $=392437845017$

8. Teixeira J. Análisis acústico vocal - Determinación del Jitter y Shimer para diagnóstico de patologías del habla. Brazil: Biblioteca Digital de IPB; 2011.

Recibido: $14 / 02 / 2020$

Aceptado: 22/05/2020

Rev Hered Rehab. 2020; 1:13-19.
(Citado el 8 de Junio del 2015) Disponible en: ht t ps://bibliotecadigital.ipb.pt/ bitstream/10198/7282/1/artigo publicado.pdf

9. Monteiro M. Uma análise computadorizada espectográfica dos formantes das vagáis orais do portugués brasileiro falado em Sao Paulo. Sao Paulo: Escola Paulista de Medicina; 1996.

10.Zañartu M. Aplicaciones del análisis acústico en los estudios de la voz humana. Santiago: Universidad Perez Rosales; 2003. (Citado el 8 de Junio del 2015) Disponible en: http://www.sld.cu/galerias/pdf/sitios/ rehabilitacion-logo/aplicaciones_del_analisis_ acustico_de_la_voz_humana.pdf

11. Suárez S. Perfiles de parámetros acústicos de la Voz, su uso e incidencia como método para la implantación y rehabilitación de la Voz y el Habla. Rev. Cubana de Informática Médica; 2014. (Citado el 8 de Junio del 2015) Disponible en: http://www.rcim.sld.cu/ revista 6/articulo_htm/perfiles.htm

12. Cobeta I, Marín A. Trastornos de la voz. Barcelona: Vivir Mejor; 2015.

13. Devars F. Lesiones orgánicas laríngeas en el niño. Marsella:Marsella Solal; 1987.

14. Vila J M. Guía de Intervención Logopédica en la Disfonía Infantil. Madrid: Síntesis; 2009.

15. Le Huche y Allali. La voz: Anatomía y fisiología, patología - terapéutica. Barcelona: Masson; 1993.

16. Dinville C. Los trastornos de la voz y su reeducación. Barcelona: Masson; 1981.

17. Cooper M. Modernas técnicas de rehabilitación vocal. Buenos Aires: Editorial Médica Panamericana; 1979.

18. Unda Y. Incidencias de Disfonías en los Alumnos del Primer Grado del Colegio Particular Cristo Salvador. Tesis de Grado. Lima: Pontificia Universidad Católica del Perú; 2013. (Citado el 8 de Junio del 2015) Disponible en: http://tesis.pucp. edu.pe/repositorio/bitstream/handle/20.500.12404/ 5035/UNDA RIOS YANINA INCIDENCIAS SALVADOR.pdf? sequence $=1 \&$ isAllowed $=\mathrm{y}$

19. Borragán A, Barrio J, Guisández M, Fernández S. Sensibilización vocal de un grupo de niños y profesores. Boletín de AELFA. 1999; (2): 6-22.

20. Colombo F. Estudios Exploratorios sobre la prevalencia de disfonía infantil en niños de 8 a 12 años. Rosario: Universidad del Rosario; 1999.

21. Feijó L. Disfonía y comportamientos vocales en niños: Revisión de literatura. Tesis de Grado. Florianópolis. Universidad Federal de Santa Catarina de Brazil; 2014.

22. Núñez B, González M, Peláez G, González L, Fernández F, Morato G. Análisis acústico de la voz mediante el progama Praat: estudio comparativo con el programa Dr. Speech. Acta otorrinolaringológica española. 2014; 65(3):170-176.

23. Maxell. H-MIC Detachable $3.5 \mathrm{~mm}$ Neckband Headset. Panama: Maxell; 2015. 